\title{
An alternating direction (ADI) method for a self-acting rectangular gas bearing
}

\author{
R. W. DE VRIES \\ Department of Applied Mathematics, Twente University of Technology, Enschede, the Netherlands
}

(Received June 6, 1974)

\section{SUMMARY}

The stationary Reynolds equation is solved over a rectangular region. The problem is linearized by Picard linearization. The ADI method is used to solve the resulting set of linear equations. A set of parameters is introduced to speed up convergence as well for the Picard linearization as for the ADI method. A comparison is made with Booy-Coleman's method. Results are given for bearing numbers 10 to 1000 .

\section{Introduction}

In gas-bearing problems the so-called Reynolds equation has to be solved numerically, for details see [1]. In this equation the ratio between viscous and non-viscous forces is given by the bearing number $A$.

For low and moderate values of $\Lambda$ many numerical methods iterative and direct are available. For large values of $A(\Lambda>100)$ computing time increases and direct methods are preferred to iterative methods. A commonly used direct method is the Booy-Coleman method $[2,3]$. This method is rather complex in programming. It needs a large memory space and with increasing $A$ the number of orthogonalizations increases and so the computing time.

Although the ADI method is not a new approach in gas-bearing problems $[1,5]$ the method here described uses a set of parameters to accelerate convergence. The acceleration parameters for the ADI method are so chosen that it, reduces to a direct method. In comparison with the Booy-Coleman method the here described method is simple to program, uses less memory and is, especially for high values of $A$ more efficient.

Computations are made for $A$ in the range 10 to 1000 , although it is not necessary for $A$ to be in this range. In the computations the number of mesh points varies from about 100 to 1000 .

\section{Analysis of the problem}

Figure 1 gives a sketch of a rectangular gas bearing with bearing width $B$ and bearing length $L$. The sliding velocity is assumed to be $-\boldsymbol{i} U_{0}$, where $\boldsymbol{i}$ indicates a positive unit vector.

We assume in the bearing viscous, isothermal and laminar flow so the density $\rho(X, Y)$ is proportional to the pressure $P(X, Y)$, and the viscosity $\sigma$ is constant. From Fig. 1 it follows that $H=\left(H_{0} / X_{0}\right) X$, if we assume the stationary case. The pressure $P(X, Y)$ can be calculated by solving the Reynolds equation for $P$.

This equation reads in the instationary case $[1]$ :

$$
\operatorname{div}\left\{\frac{\rho H^{3}}{12 \sigma} \operatorname{grad} P-\frac{1}{2} \rho H U\right\}-\frac{\partial}{\partial t} \rho H=0 .
$$

In our case it reduces to

$$
\frac{1}{12 \sigma} \operatorname{div}\left(P H^{3} \operatorname{grad} P\right)+\frac{U_{0}}{2} \frac{\partial(P H)}{\partial X}=0
$$

with $P=P_{a}$ as the boundary condition, where $P_{a}$ is the ambient pressure. 


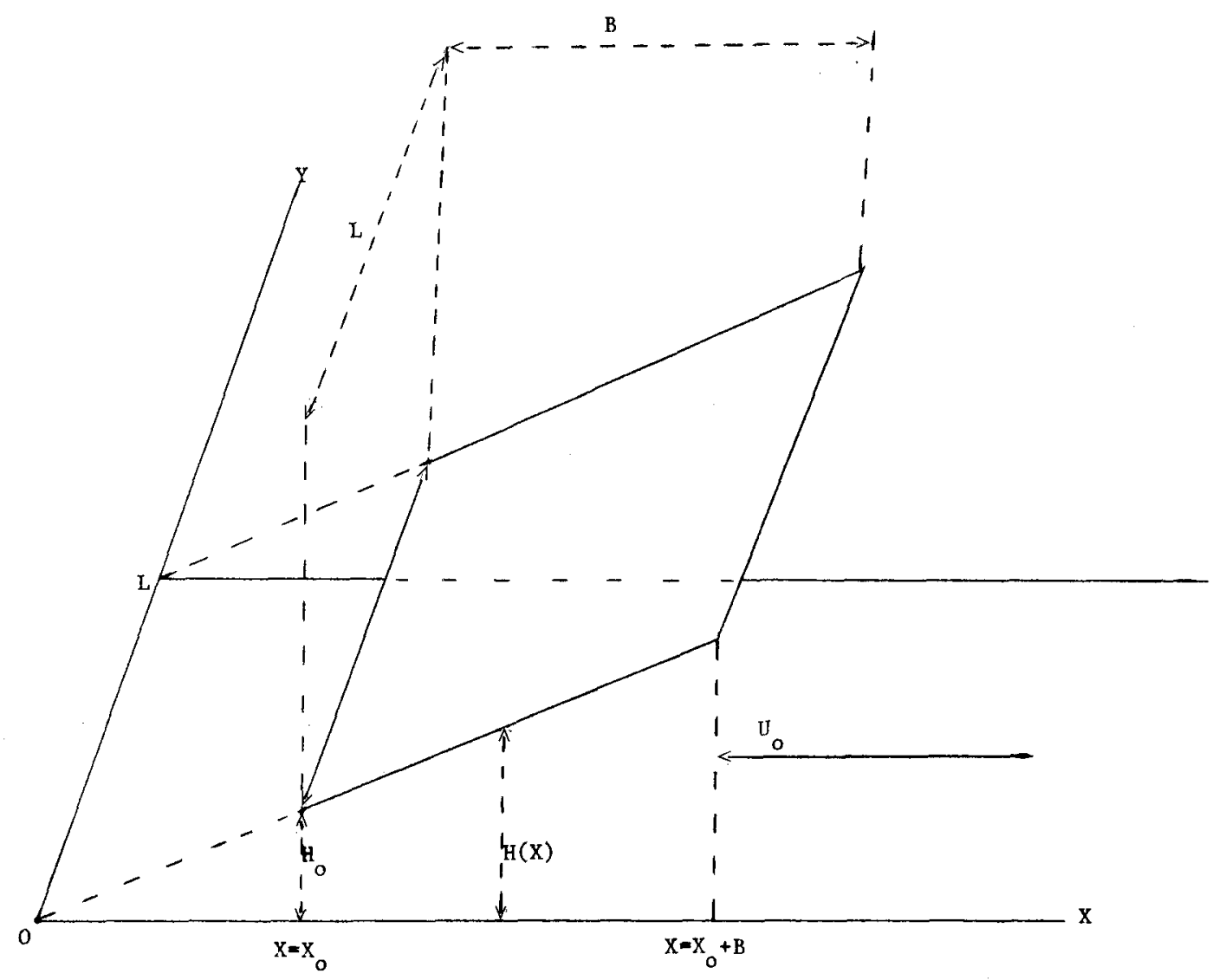

Figure 1. Plane rectangular slider bearing schematic.

Introducing the dimensionless quantities

$$
p=\frac{P}{P_{a}}, \quad x=\frac{X}{X_{0}}, \quad y=\frac{Y}{X_{0}}, \quad h=\frac{H}{H_{0}},
$$

the Reynolds equation reduces to:

$$
\frac{\partial}{\partial x}\left(p x^{3} \frac{\partial p}{\partial x}\right)+\frac{\partial}{\partial y}\left(p x^{3} \frac{\partial p}{\partial y}\right)+6 \frac{U_{0} \sigma X_{0}}{H_{0}^{2} P_{a}} \frac{\partial(p x)}{\partial x}=0 .
$$

Using the definition $Q=p^{2} x^{2}$ and $A=6 U_{0} \sigma X_{0} /\left(H_{0}^{2} P_{a}\right)$, the Reynolds equation can be written

$$
\frac{\partial^{2} Q}{\partial x^{2}}+\frac{\partial^{2} Q}{\partial y^{2}}-\frac{1}{x} \frac{\partial Q}{\partial x}=-\frac{\Lambda}{x Q^{\frac{2}{2}}} \frac{\partial Q}{\partial x}
$$

with boundary conditions:

$$
\begin{aligned}
& Q(1, y)=1 \\
& \left.Q\left(1+\frac{B}{X_{0}}, y\right)=\left(1+\frac{B}{X_{0}}\right)\right\} \quad 0 \leqq y \leqq L / 2, \\
& Q(x, 0)=x^{2} \\
& \left.Q_{y}\right|_{y=L / 2}=0 \\
& 1 \leqq x \leqq 1+B / X_{0}
\end{aligned}
$$

where $Q_{y}$ indicates differentiation with respect to $y$. The condition $\left.Q_{y}\right|_{y=L / 2}=0$ is a consequence of the symmetry of the problem. 


\section{Description of the method of solution}

In order to solve the problem use will be made of an iterative method with some special features. Therefore instead of the non-linear equation (1) we consider the linearized equation :

$$
Q_{x x}^{(k)}+Q_{y y}^{(k)}+(-1 / x+\mu) Q_{x}^{(k)}-\omega Q^{(k)}=Q_{x}^{(k-1)}\left(\frac{-\Lambda}{x\left(Q^{(k-1)}\right)^{\frac{1}{2}}}+\mu\right)-\omega Q^{(k-1)}
$$

with $k=1,2,3, \ldots$, and $Q_{x}$ and $Q_{y}$ etc. indicates differentiation with respect to $x$ and $y$, respectively. Estimates for the starting value $Q^{(0)}$ and the parameters $\mu$ and $\omega$ will be given in the sequel.

Discretizing with the normal 5-point molecule gives

$$
\begin{aligned}
& Q_{x x}=\frac{Q_{i-1, j}-2 Q_{i, j}+Q_{i+1, j}}{h_{x}^{2}}+O\left(h_{x}^{2}\right), \\
& Q_{y y}=\frac{Q_{i, j-1}-2 Q_{i, j}+Q_{i, j+1}}{h_{y}^{2}}+O\left(h_{y}^{2}\right), \\
& Q_{x}=\frac{Q_{i+1, j}-Q_{i-1, j}}{2 h_{x}}+O\left(h_{x}^{2}\right),
\end{aligned}
$$

In Fig. 2 the meshsize and the number of meshpoints is given.

Defining $\alpha=2(1+\gamma)+\omega h_{x}^{2}$, and $\beta_{i}=h_{x}\left(\mu-1 / x_{i}\right) / 2$, gives for the discretized form of equation (2):

$$
\left(-1+\beta_{i}\right) Q_{i-1, j}^{(k)}+\alpha Q_{i, j}^{(k)}+\left(-1-\beta_{i}\right) Q_{i+1, j}^{(k)}-\gamma Q_{i, j-1}^{(k)}-\gamma Q_{i, j+1}^{(k)}=-h_{x}^{2} U_{i, j}^{(k-1)}
$$

with $\gamma=h_{x}^{2} / h_{y}^{2}$ and $i=1(1) M-2, j=0(1) N-2$,

$$
U_{i, j}^{(k-1)}=\left(\frac{-\Lambda}{x_{i}\left(Q_{i, j}^{(k-1)}\right)^{\frac{1}{2}}}+\mu\right)\left(\frac{Q_{i+1, j}^{(k-1)}-Q_{i-1, j}^{(k-1)}}{2 h_{x}}\right)-\omega Q_{i, j}^{(k-1)}
$$

The boundary conditions become:

$$
\begin{aligned}
& Q(0, j)=1, \\
& Q(i, N-1)=x_{i}^{2}, \\
& Q(M-1, j)=\left(1+\frac{B}{X_{0}}\right)^{2},
\end{aligned}
$$

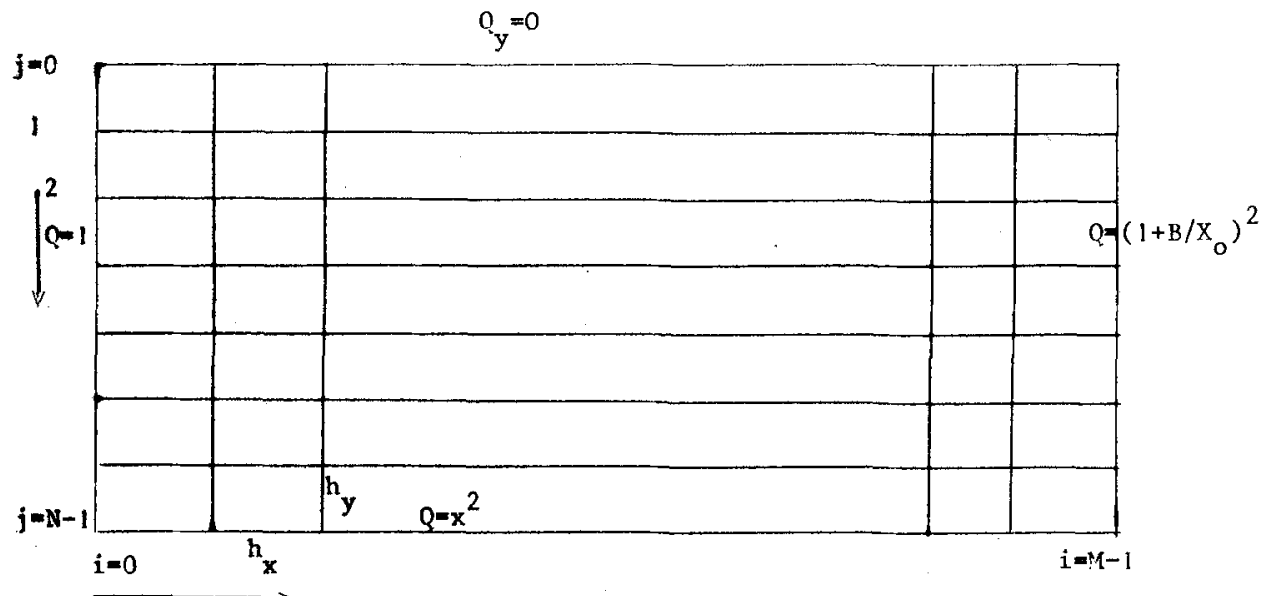

Figure 2. The rectangular integration region, $h_{x}$ and $h_{y}$ sides of the rectangular meshes. $N=$ number of horizontal lines. $N=$ number of vertical lines. 
and $Q(i, 1)=Q(i,-1)$.

The discretized problem gives rise to a set of linear equations $A Q=F$.

The matrix $A$ is a sparse matrix and the non-zero elements can be composed by the following rules:

$$
\begin{array}{ll}
a_{i i}=\alpha, & i=1,2, \ldots(N-1)(M-2), \\
a_{i, i+1}=-\gamma, & i=2,3, \ldots, N-1, N+1, \ldots, 2 N-2,2 N, \ldots(N-1)(M-2)-1, \\
a_{i, i+1}=-2 \gamma, & i=1, N, 2 N-1,3 N-2, \ldots,(M-3)(N-1)+1, \\
a_{i, i-1}=-\gamma, & i=2,3, \ldots N-1, N+1, \ldots, 2 N-2,2 N, \ldots(N-1)(M-2), \\
a_{i, i-1}=0, & i=N, 2 N-1,3 N-2, \ldots,(M-3)(N-1)+1, \\
a_{i, N+i-1}=-1-\beta_{p}, & i=1,2, \ldots(N-1)(M-3) \text { and } p=\operatorname{entire}\left(\frac{i-1}{N-1}+1\right), \\
a_{i+1, i-N+2}=-1+\beta_{p}, & i=N-1, N, N+1, \ldots,(N-1)(M-2)-1
\end{array}
$$

All the other elements are zero and $\boldsymbol{F}$ is defined by:

$$
\boldsymbol{F}=\left[\begin{array}{l}
-h_{x}^{2} U_{1,0}+1-\beta_{1} \\
-h_{x}^{2} U_{1,1}+1-\beta_{1} \\
\vdots \\
-h_{x}^{2} \dot{U}_{1, N-1}+1-\beta_{1}-x_{1}^{2} \gamma \\
-h_{x}^{2} U_{2,0} \\
\vdots \\
-h_{x}^{2} U_{2, N-1}+x_{2}^{2} \gamma \\
\vdots \\
-h_{x}^{2} U_{M-2, N-1}+x_{M-2}^{2} \gamma+\delta
\end{array}\right]
$$

$$
\delta=\left(1+\beta_{M-2}\right)\left(1+\frac{B}{X_{0}}\right)^{2} .
$$

According to the ADI method [4] the matrix $A$ is splitted in the matrices $\bar{H}$ and $\bar{V}$, so $A=\bar{H}+\bar{V}$, where

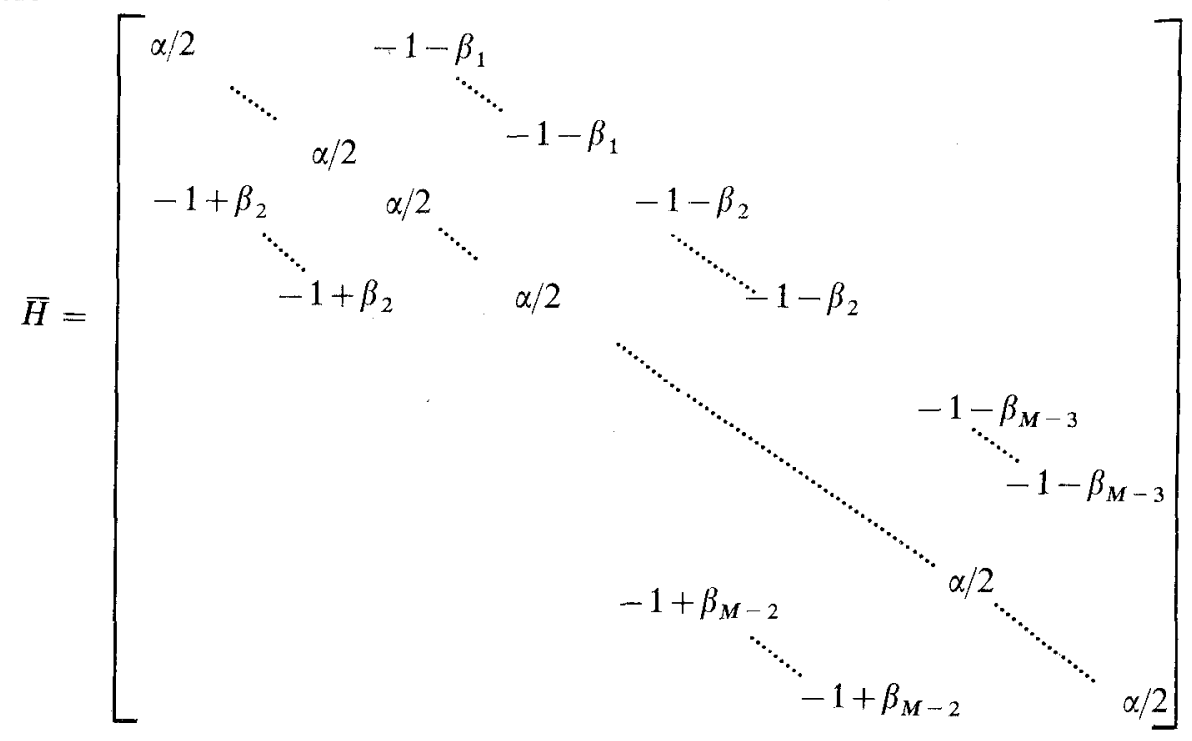

The blocksize of the blocks in $\bar{H}$ is $N-1$. 
If we define the matrix $W$ to be:

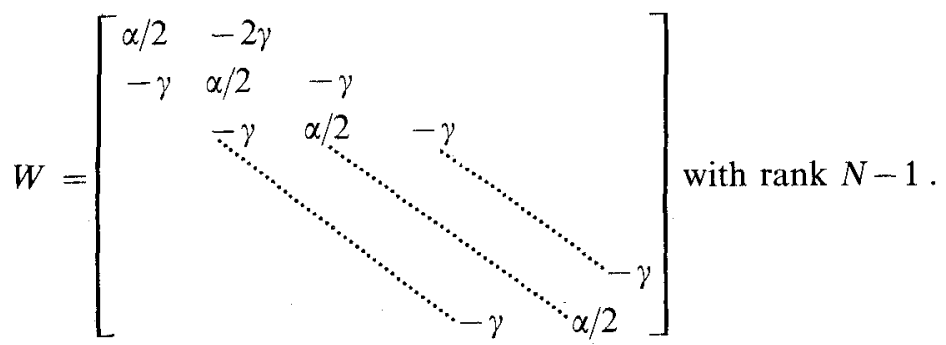

then $\bar{V}$ can be written as a diagonal block matrix :

$$
\widetilde{V}=\left[\begin{array}{lllll}
W & & & & \\
& W & & & 0 \\
& & W & & \\
& & \cdot & \\
& & & \cdot & \\
0 & & & \cdot & \\
& & & & W
\end{array}\right]
$$

So the $M-2$ uncoupled matrices $W$ compose the matrix $\bar{V}$.

In the ADI method a set of acceleration parameters has to be chosen. Calling these parameters $r_{m}$ then the system $A Q=F$ can be solved with the following iterative scheme [4]:

$$
\left.\begin{array}{l}
\left(\bar{H}+r_{m} I\right) Q^{\left(s+\frac{1}{2}\right)}=\left(r_{m} I-\bar{V}\right) Q^{(s)}+F \\
\left(\bar{V}+r_{m} I\right) Q^{(s+1)}=\left(r_{m} I-\bar{H}\right) Q^{\left(s+\frac{1}{2}\right)}+F
\end{array}\right\}
$$

The so-called Peaceman-Rachford matrix is defined by:

$$
T_{r}=(\bar{V}+r I)^{-1}(r I-\bar{H})(\bar{H}+r I)^{-1}(r I-\bar{V})
$$

and the error in the $s$ th iterationstep by $\varepsilon^{(s)}=Q^{(s)}-Q$. So

$$
\varepsilon^{(s)}=\prod_{p=1}^{s} T_{r_{p}} \varepsilon^{(0)}
$$

In linearizing equation (1) we have chosen for the so-called Picard linearization.

By considering equation (2), it is seen that the matrices $-1 / h_{x}^{2} \bar{H}$ and $-1 / h_{y}^{2} \bar{V}$ are the discretized forms of the operators

$$
\frac{\partial^{2}}{\partial x^{2}}+(-1 / x+\mu) \frac{\partial}{\partial x}-\omega / 2 \text { and } \frac{\partial^{2}}{\partial y^{2}}-\omega / 2 .
$$

By direct calculation it follows that these operators do not commute if $\mu=\mu(x, y)$ or $\omega=\omega(x, y)$. A sufficient condition for the matrices $\bar{H}$ and $\bar{V}$ to commute is that $\omega$ and $\mu$ are constants. It is easy to see that if the matrices $\bar{H}$ and $\bar{V}$ commute, the following matrices commute:

$$
(\bar{V}+r I)^{-1},(r I-\bar{H}),(\bar{H}+r I)^{-1} \text { and }(r I-\bar{V}) .
$$

Now we are able to look for a set of acceleration parameters $\left\{r_{m}\right\}$. Suppose $\bar{V}$ with rank $\mathscr{M}$ possesses a set of $\mathscr{M}$ linear independent eigenvectors $\left\{\boldsymbol{t}_{k}\right\}$ and corresponding eigenvalues $\left\{v_{k}\right\}$. Then

or

$$
\boldsymbol{\varepsilon}^{(0)}=\sum_{k=1}^{\mathscr{M}} \alpha_{k} \boldsymbol{t}_{k}, \quad \boldsymbol{\varepsilon}^{(s)}=\prod_{j=1}^{s} T_{r_{j}} \sum_{k=1}^{\mathscr{M}} \alpha_{k} \boldsymbol{t}_{k}
$$

with

$$
\boldsymbol{\varepsilon}^{(s)}=\sum_{k=1}^{\mathscr{M}} \alpha_{k} \prod_{j=1}^{s} T_{r_{j}} \boldsymbol{t}_{k}=\sum_{k=1}^{\mu h} \alpha_{k} \tilde{H} \boldsymbol{t}_{k} \prod_{j=1}^{s} \frac{r_{j}-v_{k}}{r_{j}+v_{k}}
$$




$$
\tilde{H}=\left(r_{s} I-\bar{H}\right)\left(\bar{H}+r_{s} I\right)^{-1}\left(r_{s-1} I-\bar{H}\right)\left(\bar{H}+r_{s-1} I\right) \ldots\left(r_{1} I-\bar{H}\right)\left(\bar{H}+r_{1} I\right)^{-1} .
$$

The following inequality holds:

$$
\left\|\varepsilon^{(s)}\right\| \leqq \sum_{k=1}^{\mathscr{M}}\left|\alpha_{k}\right|\left\|\tilde{H} t_{k}\right\| \prod_{j=1}^{S} \frac{r_{j}-v_{k}}{r_{j}+v_{k}} .
$$

By choosing $r_{j}=v_{j},\left\|\varepsilon^{(s)}\right\|=0$ after $\mathscr{M}$ iterations.

$\bar{V}$ is completely reducible and is composed of $M-1$ matrices $W$ with rank $N-1$. The iteration scheme (3) reduces after $N-1$ iterations to a direct method. To get the above mentioned method we have to calculate the eigenvalues of $W$. Instead of $W$, we consider the matrix $\widetilde{W}$,

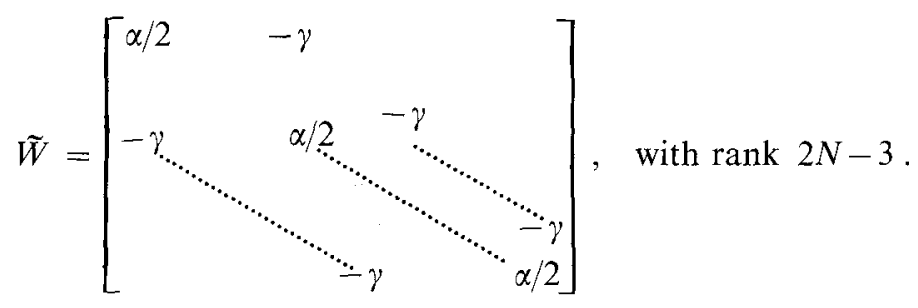

It is easy to see that the $k$ th component of the $q$ th eigenvector of $W$ can be written as $d \sin [q \Pi k /(2 N-2)]$, with $d$ a constant $(k=1,2, \ldots, 2 N-3)$.

The first row of $W$ is situated at the $(N-1)$ th row of $\tilde{W}$. So we are looking for eigenvectors of $\tilde{W}$ with components $d \sin [q \Pi k /(2 N-2)]$ with $k=N-1, N, \ldots, 2 N-3$. Substitution of the so formed eigenvectors $t$ in the matrix vector product $W t$, gives as eigenvalues for $W$,

$$
-2 \gamma \cos \frac{q \Pi}{2 N-2}+\alpha / 2
$$

with $q=2 p-1$ and $p=1(1) N-1$.

\section{Estimates for $\omega$ and $\mu$}

In equation (1) the parameters $\mu$ and $\omega$ are introduced to speed up the convergence of problem (2).

We define $z^{(k)}=Q^{(k)}-Q^{(k-1)}, k \geqq 2$. Using the definition

$$
C(k)=\frac{-1}{x\left(Q^{(k)}\right)^{\frac{1}{2}}}+\mu,
$$

it follows from (2) that

$$
z_{x x}^{(k)}+z_{y y}^{(k)}+(\mu-1 / x) z_{x}^{(k)}-\omega z^{(k)}=C(k-1) Q_{x}^{(k-1)}-C(k-2) Q_{x}^{(k-2)}-\omega z^{(k-1)},
$$

with $z=0$ on the boundary of the rectangle $G\left\{x, y \mid 1 \leqq x \leqq 1+B / X_{0}, 0 \leqq y \leqq L\right\}$. We expand $\left(Q_{x} /(Q)^{\frac{1}{2}}\right)^{(k-1)}$ in the function space $\left\{Q^{(k)}\right\}$ about the "point" $Q^{(k-2)}$, neglecting second order terms. This gives:

$$
z_{x x}^{(k)}+z_{y y}^{(k)}+(\mu-1 / x) z_{x}^{(k)}-\omega z^{(k)}=C(k-2) z_{x}^{(k-1)}+\left(\frac{A Q_{x}^{(k-2)}}{2 x Q^{(k-2)}\left(Q^{(k-2)}\right)^{\frac{1}{2}}}-\omega\right) z^{(k-1)} .
$$

This equation is an elliptic equation which follows the maximum principle if $\omega>0$ [7, p. 88]. So

$$
\max _{G}\left|z^{(k)}\right| \leqq K \max _{G}\left(C(k-2) z_{x}^{(k-1)}+\left(\frac{A Q_{x}^{(k-2)}}{2 x Q^{(k-2)}\left(Q^{(k-2)}\right)^{\frac{1}{2}}}-\omega\right) z^{(k-1)}\right) .
$$

As $Q$ is bounded on $G$ if $A \rightarrow \infty$ we choose for

$$
\mu=\frac{1}{2}\left(\min _{G} \frac{A}{x\left(Q^{(k-2)}\right)^{\frac{1}{2}}}+\max _{G} \frac{A}{x\left(Q^{(k-2)}\right)^{\frac{1}{2}}}\right)=\Lambda / 2\left(1+1 /\left(1+B / X_{0}\right)^{2}\right),
$$

to minimize $C(k-2)$. 
If $A \rightarrow \infty, Q_{x}$ is not bounded everywhere, so it is not possible to get a feasible estimate for $\omega$. However if $\Lambda \rightarrow \infty$ we can make the assumption that $Q$ is independent of $y$ (see the next section) and as an estimation for $Q_{x}$ we use for $A \gg 1$, the mean value of the asymptotic value of $Q_{x}$ for $A \rightarrow \infty$ over the interval $1 \leqq x \leqq 1+B / X_{0}$. So

$$
\omega=A / 4\left(1+\left(1+B / X_{0}\right)^{2}\right) /\left(B / X_{0}\right)\left(1+1 /\left(1+B / X_{0}\right)^{4}\right) .
$$

\section{Estimation of the starting value $Q^{(0)}$}

A commonly used estimate for $Q^{(0)}$ is $Q^{(0)}=x^{2}$. This is a feasible estimation for small values of the bearing number $A$. For large values of $A$ we assume that, except for a boundary layer $0 \leqq y \leqq \delta, Q$ is independent of $y$.

Using the definition $B_{0}=\left(1+B / X_{0}\right), Q$ tends to $B_{0}^{2}$ if $A$ tends to infinity, and equation (1) is approximated by

$$
Q_{x x}=-\Lambda /\left(B_{0}^{2}\right) Q_{x} / x
$$

with boundary conditions $Q=1$ if $x=1$ and $Q=B_{0}^{2}$ if $x=B_{0}$.

Solving this equation gives

$$
Q=Q_{b}=\frac{\left(B_{0}^{2}-1\right)\left(x^{-\Lambda / B_{0}+1}-1\right)}{B_{0}^{-A / B_{0}+1}-1}+1
$$

In the boundary layer (1) is approximated by $Q_{y y}=-2 A / B_{0}$ and the boundary conditions are

$$
\begin{aligned}
& Q_{y}=0 \\
& Q=Q_{b}
\end{aligned} \quad y=\delta, \text { and } Q=x^{2} \text { if } y=0
$$

So we get

$$
Q=-\Lambda / B_{0} y^{2}+2 \Lambda \delta / B_{0} y+x^{2}
$$

and $\delta=\left(B_{0}\left(Q_{b}-x^{2}\right) / \Lambda\right)^{\frac{1}{2}}$.

We use (4) as a starting value if $\delta \leqq y \leqq L / 2$ and (5) if $0<y<\delta$.

\section{Numerical results}

TABLE 1

Pressure distribution for $A=10,\left(Q^{\frac{1}{2}} / x\right)-1=p$.

\begin{tabular}{llll}
\hline$X$ & $\mathrm{BC}(M=21, N=7)$ & $\mathrm{ADI}(M=21, N=7)$ & ADI $(M=41, N=13)$ \\
\hline 1.05 & 0.1520 & 0.1525 & 0.1527 \\
1.10 & 0.2176 & 0.2183 & 0.2184 \\
1.15 & 0.2441 & 0.2449 & 0.2450 \\
1.20 & 0.2504 & 0.2513 & 0.2512 \\
1.25 & 0.2454 & 0.2464 & 0.2463 \\
1.35 & 0.2194 & 0.2205 & 0.2204 \\
1.40 & 0.2029 & 0.2040 & 0.2039 \\
1.45 & 0.1854 & 0.1866 & 0.1864 \\
1.50 & 0.1676 & 0.1688 & 0.1687 \\
1.55 & 0.1499 & 0.1511 & 0.1510 \\
1.60 & 0.1323 & 0.1335 & 0.1334 \\
1.65 & 0.1150 & 0.1162 & 0.1161 \\
1.70 & 0.0979 & 0.0992 & 0.0991 \\
1.75 & 0.0811 & 0.0824 & 0.0823 \\
1.80 & 0.0645 & 0.0658 & 0.0657 \\
1.85 & 0.0480 & 0.0493 & 0.0493 \\
1.90 & 0.0316 & 0.0329 & 0.0329 \\
1.95 & 0.0152 & 0.0165 & 0.0165 \\
\hline
\end{tabular}


The calculations were made on the IBM 360/50 computer of the T.H. Twente. The results of this method are compared with the results of the Booy-Coleman method. In the following tables the pressure computed by Booy-Coleman (BC) and ADI are shown for the line $y=L / 2$, and $\Lambda=10$ and 100 .

The programs are written in Fortran, using single precision for the Booy-Coleman method and double precision for the ADI method.

The dimensions are taken $L=B=X_{0}=1$.

TABLE 2

Pressure distribution for $A=100$.

\begin{tabular}{lllll}
\hline$X$ & BC $(M=21, N=7)$ & ADI $(M=21, N=7)$ & ADI $(M=41, N=7)$ & ADI $(M=81, N=7)$ \\
\hline 1.05 & 0.9331 & 0.9352 & 0.8484 & 0.8363 \\
1.10 & 0.7981 & 0.8003 & 0.8004 & 0.7986 \\
1.15 & 0.7246 & 0.7266 & 0.7263 & 0.7260 \\
1.20 & 0.6543 & 0.6561 & 0.6561 & 0.6561 \\
1.25 & 0.5896 & 0.5913 & 0.5913 & 0.5913 \\
1.30 & 0.5299 & 0.5314 & 0.5314 & \\
1.35 & 0.4745 & 0.4758 & 0.4758 & \\
1.40 & 0.4229 & 0.4241 & 0.4241 & \\
1.45 & 0.3748 & 0.3759 & 0.3759 & \\
1.50 & 0.3298 & 0.3308 & 0.3308 & \\
1.55 & 0.2876 & 0.2885 & 0.2885 & \\
1.60 & 0.2480 & 0.2487 & 0.2487 & \\
1.65 & 0.2106 & 0.2112 & 0.2112 & \\
1.70 & 0.1754 & 0.1759 & 0.1759 & \\
1.75 & 0.1421 & 0.1425 & 0.1425 & \\
1.80 & 0.1106 & 0.1109 & 0.1109 & \\
1.85 & 0.0807 & 0.0810 & 0.0810 & \\
1.90 & 0.0524 & 0.0526 & 0.0526 & \\
1.95 & 0.0255 & 0.0256 & 0.0256 &
\end{tabular}

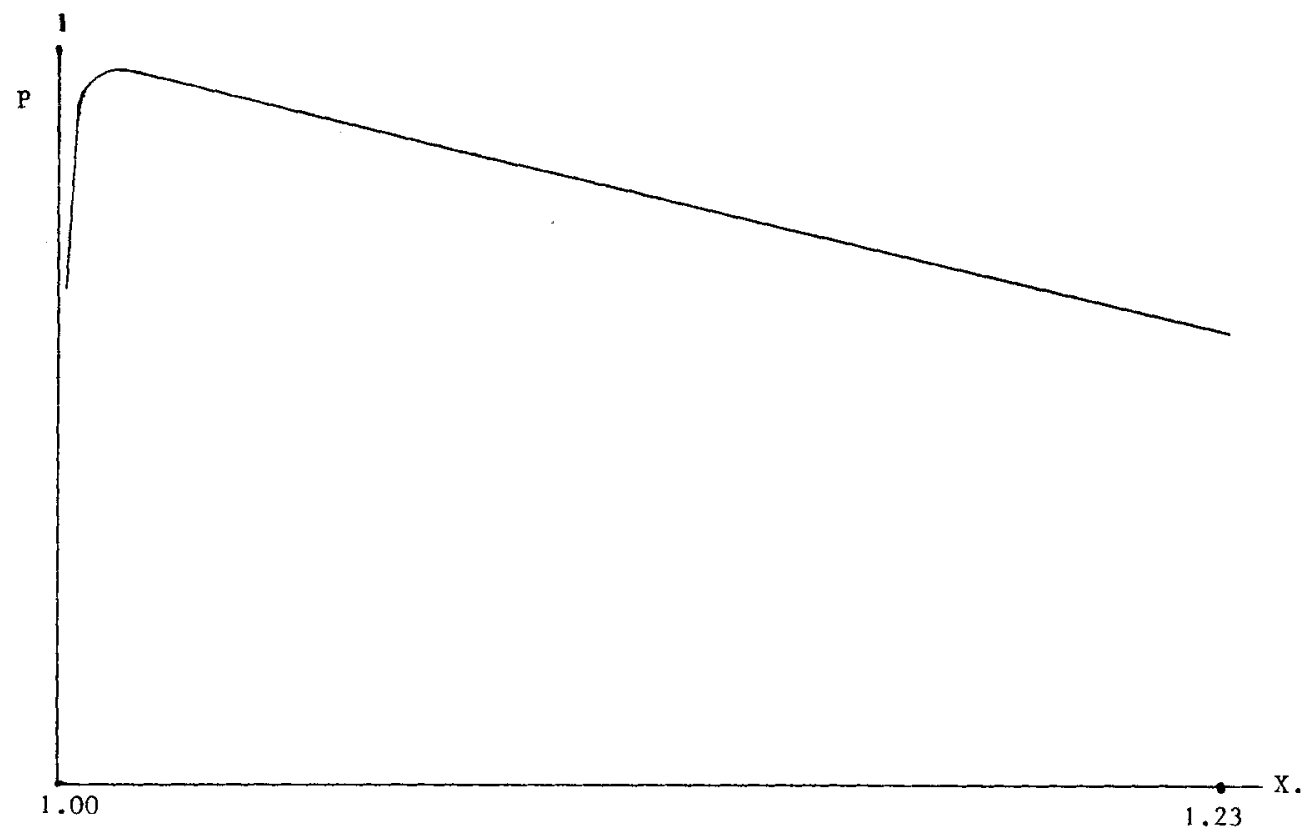

Figure 3. Pressure distribution for $A=1000$. 
In the Booy-Coleman method the nonlinear problem was linearized with the so-called quasi-linearization. So convergence was quadratic. Number of iterations for $\mathrm{BC}$ was 2 for ADI 10.

Computing time was slightly in favour of ADI, $24 \mathrm{sec}$. to $28 \mathrm{sec}$. CPU for BC. For higher values of $A$, the computing time was highly in favour of ADI, although the ADI computations are in double precision.

In Table 2 we see the pressure distribution for $A=100$, computing time for $\mathrm{BC} 101 \mathrm{sec}$. CPU, for ADI resp. $40 \mathrm{sec}$., $67 \mathrm{sec}$. and $191 \mathrm{sec}$. CPU.

From Table 2 we see that it is difficult to get accurate results near $x=1$ for higher values of $A$. Because $x>1$,

$$
x^{-A /\left[1+\left(B / X_{0}\right)\right]+1} \rightarrow 0 \text { if } \Lambda \rightarrow \infty .
$$

In the program, a test is made if

$$
\left(1+\delta_{x}\right)^{-\boldsymbol{A} /\left[1+\left(B / X_{0}\right)\right]+1}=10^{-10}
$$

and $\delta_{x}>10^{-4}$. If true then $5 \delta_{x}$ is used as integration interval in the $x$-direction. For $x>5 \delta_{x}$ we use the solution $Q=\left(1+B / X_{0}\right)^{2}$. Now we are able to get accurate results near $x=1$ for high values of $A$, without increasing the number of mesh points.

The pressure distribution for $A=1000, \delta_{x}=0.047$ is shown in Fig. 3. Only the interval $1 \leqq x \leqq 1.23$ is plotted, for $x>1.23$ we use the solution $Q=4$.

The author is indebted to Prof. P. J. Zandbergen, Mr. van Beckum and Mr. van Eck of this institute.

\section{REFERENCES}

[1] V. Castelli and J. Pirvics, Review of Numerical Methods in Gas Bearing Film Analysis, Journal of Lubrication Technology, Trans. ASME, Series F, 90, 4 (1968) 777-792.

[2] M. L. Booy, A Non iterative Solution of Poisson's and Laplace's Equations With Application to Slow Viscous Flow, Journal of basic Engineering, Trans. ASME, Series D, 88, 4 (1966) 725-733.

[3] R. Coleman, The Numerical Solution of Linear Elliptic Equations, Journal of Lubrication Technology, Trans. ASME, Series F, 90, 4 (1968) 773-776.

[4] R. S. Varga, Matrix Iterative Analysis, Prentice Hall, New York (1962).

[5] I. C. Tang, The Design and Analysis of Rectangular Gas Slider Bearings, Wear, 20 (1972) 59-65. 4.

[6] J. Douglas, jr., Alternating Direction Iteration for Mildly Nonlinear Elliptic Difference Equations, Numerische Math., 3 (1961) 92-98.

[7] A. Friedman, Partial Differential Equations, Holt, Rinehart and Winston Inc. (1969). 